\title{
Water on the Moon
}

\author{
Yvonne J. Pendleton \\ NASA Ames Research Center \\ Solar System Exploration Research Virtual Institute, Director \\ Mail Stop 17-1, Moffett Field, CA 94035 \\ email: yvonne.pendleton@nasa.gov
}

\begin{abstract}
After years of thinking the Moon is dry, we now know there are three manifestations in which water appears on the Moon today: 1) Previously hypothesized buried deposits of volatiles at the lunar poles were found at Cabeus crater. There are questions about the origin of such volatiles (i.e., in-falling comets \& meteorites, migration of recently formed surficial $\mathrm{OH} / \mathrm{H}_{2} \mathrm{O}$, and accumulated release from the interior), but there is no doubt the water is there. 2) Widespread, thinly-distributed, surficial $\mathrm{OH}$ ( or $\mathrm{H}_{2} \mathrm{O}$ ) has been clearly detected across all types of lunar terrain. The consensus is that the $\mathrm{OH}$ is derived from solar wind, but we do not know how quickly it forms, nor how mobile it is. 3) The amount of water present soon after the Moon formed is now documented in new analyses of lunar materials in volcanic glass beads, apatites and plagioclase feldspars. Apollo era sample analyses were not precise enough to distinguish between indigenous lunar water and terrestrial contamination. Measurements with modern equipment are more precise (both elemental and isotopic), and can better constrain a host of processes (e.g. diffusion, thermal cycling). Scientists around the world are studying lunar water. Ongoing analyses are informing a number of hypotheses and theories about the connection between the Earth and its wet Moon.
\end{abstract}

Keywords. water, Moon, SSERVI, NLSI, lunar science

\section{Introduction}

Together with the broader lunar science community, researchers at NASA's Solar System Exploration Research Virtual Institute (SSERVI) and its predecessor, the NASA Lunar Science Institute (NLSI), have made tremendous progress investigating the presence of water on the Moon. This high level summary brings together a number of key discoveries and concepts which would not have been possible without new technologies and the recent cadre of spacecraft sent to the Moon by several countries. Research using data from these missions has added immensely to our understanding of the Moon, from its formation to present day activities. This is an important time in lunar science, where novel techniques are yielding tremendous insights into the Moon's past, in turn shedding light on the history of our own planet.

\section{Lunar water in polar cold traps}

Despite much discussion to the contrary, the literature supports the theoretical existence of water at the coldest points on the lunar surface, where water is far more stable than the noble gases or other possible constituents of the lunar atmosphere (Watson et al. 1961). Possible mechanisms for delivery to the surface include: arrival via impacts, migration of surficial $\mathrm{OH}$ or $\mathrm{H}_{2} \mathrm{O}$ from lower latitudes to the poles, and internal sources that make their way to the surface. Regardless of how it got there, the presence of water was validated by NASA's Lunar Cratering Observation and Sensing Satellite (LCROSS) 
mission. LCROSS impacted Cabeus Crater near the lunar south pole, creating a plume that was observed by the shepherding spacecraft (Colaprete et al. 2012). A combination of dirty ice grains and more pure ice grains was seen in the ejecta plume up to four minutes after impact (Colaprete et al. 2010). LCROSS found water concentrations of $6.3 \pm$ 1.6 percent by mass in Cabeus crater (Strycker et al. 2013), although Elphic et al. (2011) point out that volatile abundances of $5 \mathrm{wt} \%$ or more, distributed uniformly and homogeneously throughout Cabeus do not agree with orbital measurements, suggesting this may be a local, rather than regional, amount of water. LCROSS mission payload details can be found in Ennico et al. (2012) and a thorough summary of plume evolution and physical causes in Heldmann et al. (2015).

Prior to LCROSS and NASA's continuing Lunar Reconnaissance Orbiter (LRO) mission, launched together in 2009, NASA's Lunar Prospector (LP) Mission mapped the Moon's surface composition and looked for possible deposits of polar ice, measured magnetic and gravity fields, and studied lunar outgassing. Launched in 1998, LP carried a neutron spectrometer that detected hydrogen at both lunar poles. The data indicated that a large quantity of water ice, about 300 million tons, was mixed in the regolith at each pole. LP's neutron spectrometer measured regions where epithermal neutron flux from the surface is suppressed, indicating enhanced hydrogen content (Feldman et al. 1998). The Lunar Exploration Neutron Detector instrument on LRO mapped the hydrogen distribution over the lunar surface with spatial resolution of $10 \mathrm{~km}$ on the poles, further characterizing the neutron component of the lunar radiation environment (Mitrofanov et al. 2010). These missions provided the stepping stones to deeper understanding about the lunar volatile inventory.

\section{Surficial $\mathrm{OH} /$ water}

Launched in 2008, India's Chandrayaan-1 Mission carried the Moon Mineralogy Mapper $\left(\mathrm{M}^{3}\right)$, a U.S. instrument which revealed a widespread 2.8-3 $\mu \mathrm{m}$ absorption feature that progressively intensified from about 60 degrees poleward in both directions (Pieters et al. 2009, McCord et al. 2011). These data indicated $\mathrm{OH} / \mathrm{H}_{2} \mathrm{O}$, an unexpected finding given the high daytime temperatures in the mid-latitude regions, and the early Apollo sample sudies that indicated a dry Moon. Fortunately, two additional sources of independent observations were available from NASA's Cassini and EPOXI spacecraft. The Visual and Infrared Mapping Spectrometer (VIMS), carried aboard Cassini, flew past the Moon on its way to Saturn in 1999, and the earlier data were investigated in light of the $\mathrm{M}^{3}$ discovery. EPOXI had been making numerous close approaches to the EarthMoon system and was observing the Moon as a calibration source in the same wavelength range. Prompted by the $\mathrm{M}^{3}$ discovery, EPOXI observed the Moon in June 2009. In both cases, the $3 \mu \mathrm{m}$ lunar spectral signature was observed and confirmed (Clark et al. 2009, \& Sunshine et al. 2009), respectively. Furthermore, the EPOXI observations suggested possible diurnal effects, given the reduction in surficial $\mathrm{OH}$ as regions rotate into the Sun.

The solar wind is the likely hydroxylation source as it is composed of protons and electrons streaming at $400 \mathrm{~km} / \mathrm{sec}$, with temperatures near $10^{5} \mathrm{~K}$. Recent work in this area reveals the complexities involved in these processes, as the solar wind can both create and destroy $\mathrm{OH}$ /water on the lunar surface (Dyar et al. 2010, Farrell et al. 2015). Investigations of the mobility of $\mathrm{H}$ on the surface (Farrell et al. 2013), plus the complex nature of solar wind $\mathrm{H}$-implantation (Farrell et al. 2015) and $\mathrm{OH}$ formation processes proposed by Poston et al. (2012), are leading to greater understanding of the origin and evolution of $\mathrm{OH}$ (and possibly $\mathrm{H}_{2} \mathrm{O}$ ) on the lunar surface. One interesting result from modeling efforts is that defect properties of a crystal have as much (or more) 
control on retention in an exposed regolith as temperature and/or solar wind flux. Solar wind impacts rough up the surface, possibly removing existing $\mathrm{H}$ or $\mathrm{OH}$, but that in turn enables new $\mathrm{H}$ or $\mathrm{OH}$ implantation to occur more readily (Farrell et al. 2015). Furthermore, the lunar surface is oxide-rich $\left(\mathrm{SiO}_{2}, \mathrm{TiO}_{2}, \mathrm{FeO}_{2}\right)$, and defects in these materials are especially important in this process (Starukhina 2006, Dyar et al. 2010). The irregular and damaged fine-grained lunar soil traps solar wind protons and forms $\mathrm{OH}$. Depending on how aligned or isolated the defects are at any given time, they will either form a channel that allows enhanced diffusion, or trap the incoming particle. As the physics of these studies apply to other airless bodies, the creation and destruction of lunar $\mathrm{OH}$ holds considerable promise to understanding bodies beyond the Moon.

\section{Water from deep within the Moon}

New analyses of Apollo samples have detected magmatic water in lunar volcanic glasses of pyroclastic origin that are substantially higher than earlier studies (Saal et al. 2008, Saal et al. 2013, Hauri et al. 2011). These volcanic glasses were analyzed using microbeam methods with either SIMS or FTIR techniques which were not available when the samples were brought to Earth decades ago. The Saal and Hauri team found melt inclusions from which they could extract pre-eruptive magmatic lunar water. Trapped within olivine crystals before volcanic eruption, these melt inclusions did not experience significant post-eruptive degassing. Prior to these studies, published direct measurements of water in lunar volcanic glasses did not exceed 50 parts per million. Hauri et al. (2011) reported the lunar melt inclusions contain 615 to $1410 \mathrm{ppm}$ water and high correlated amounts of fluorine (50 to $78 \mathrm{ppm})$, sulfur (612 to $877 \mathrm{ppm})$, and chlorine (1.5 to $3.0 \mathrm{ppm})$. These volatile contents are very similar to those in primitive terrestrial mid-ocean ridge basalts and indicate that some parts of the lunar interior contain as much water as the upper mantle of the Earth. Studies of water in other lunar materials such as apatites and plagioclase feldspars (see Hauri et al. 2015 for references), plus the volcanic rock results, have provided a new and critical constraint upon the high-temperature models previously used to explain the formation and evolution of the Moon.

The Moon likely formed from a giant impact collision (Hartmann \& Davis 1975) between a roughly Mars-sized object and the proto-Earth (Canup 2004). The extreme depletion of volatiles in lunar volcanic rocks from early Apollo studies had been taken as evidence of pervasive degassing after that energetic giant impact. More recent models can explain the Earth-Moon angular momentum and early thermal history of the Moon (Cameron \& Benz 1991, Canup \& Asphaug 2001, Canup 2012, Cuk \& Stewart 2012), but they predict melting and partial vaporization of the material that enters the proto-lunar orbit, which makes it difficult to explain the newly measured abundance of volatiles in the lunar interior. However, Nakajima \& Stevenson (2015) have shown that even protolunar material vaporized by such an impact may remain gravitationally bound to the Earth and avoid escape. Latest models suggest that in the absence of escape, the Moon's observed volatile depletion could be produced during the last stages of its assembly when volatile-rich material is preferentially accreted by the Earth instead of the Moon (Canup et al. 2015). These models also predict that the inner, earlier formed portions of the Moon could be volatile rich, reflecting the composition of a potentially water-rich early Earth and/or giant impactor. Zircons as old as 4,325 Ma provide evidence for the presence of liquid water near the surface of the Earth within $230 \mathrm{Ma}$ of Earth's accretion. This observation is consistent with the conclusion that either the Earth had significant 
amounts of water before the giant impact, or the material that accreted after the giant impact (but before $4.3 \mathrm{Ga}$ ) was rich in volatiles.

\section{Summary}

Much of the paradigm-shifting work reported here was enabled by the cross-disciplinary work carried out by researchers around the world analyzing the abundance of data from the latest space missions. Our understanding of the Moon has changed significantly in the past few years. We now know there is water on the Moon; we see multiple contributorssome from long ago and some from present day. The Moon retains $\mathrm{OH}$ and water, as evidenced by material excavated from permanently shadowed regions, spectroscopic signatures on the surface, and water in lunar samples (a reminder that sample return is a gift to our future selves). The ability to process 40 year old, well-preserved, samples with new technology has enabled us to learn far more than we could originally glean from the Apollo samples. We have learned that the space environment also plays an important role in retention and/or creation of water on the surface-a process likely happening on other airless bodies. In addition, lunar resources may be key to future lunar bases and interplanetary refueling depots, given that all forms of hydrogen are useful for in situ resource utilization. The multidisciplinary teams within SSERVI are focused primarily on science questions needed for future exploration. The search for answers at the edge of our understanding will continue to benefit from the multi-institutional and international research enabled by NASA's virtual institutes.

\section{References}

Cameron, A., \& Benz, W. 1991 Icarus, 92, 204-216

Canup, R.M. 2004 Icarus, 168, 433-456

Canup, R.M. 2012 Science, 338, 1052-1055

Canup, R. M., \& Asphaug, E. 2001 Nature, 412, 708-712

Canup, R. M., Visscher, C., Salmon, J. \& Fegley, B. 2015 Nature Geoscience, in press

Clark, R. N. 2009 Science, 326, 562-564

Colaprete, A., Schultz, P., Heldmann, J., Wooden, D., \& 13 co-authors 2010, Science, 330, 463

Colaprete, A., Elphic, R., Heldmann, J., \& Ennico, K. 2012, Space Science Review,167, 23-69

Cuk, M. \& Stewart, S. T. 2012 Science, 338, 1047-1052

Dyar, M. D., Hibbitts, C. A., \& Orlando, T. M. 2010, Icarus, 208, 425-437

Elphic, R., Teodoro, L., Eke, V., \& 3 co-authors 2011 Lunar \& Planet. Sci. Conf, 2751

Ennico, K., Shirley, M., Colaprete, A., \& Osetinsky, L. 2012, Space Science Review,167, 23-69

Farrell, W. M., Hurley, D. M., \& 5 co-authors 2013, Planetary \& Space Science, 89, 15-20

Farrell, W. M., Hurley, D. M., \& Zimmerman, M. I. 2015, Icarus, 255, 116-126

Feldman, W. C., Barraclough, B. L., Maurice, S. \& 4 co-authors 1998, Science, 281, 1489

Hartmann, W. K., \& Davis, D.R. 1975 Icarus, 23, 504-515

Hauri, E., Weinreich, T., Saal, A., Rutherford, M., \& Van Orman, J. 2011, Science, 333, 213-215

Hauri, E., Saal, A., Rutherford, M., \& Van Orman, J. 2015, Earth Planet. Sci. Lett., 409, 252-264

Heldmann, J., Lamb, J., Asturias, D., Colaprete, A. \& 4 co-authors 2015, Icarus, 254, 262-275

Mitrofanov, I. G., many co-authors 2010, Science, 330, 483

McCord, T., Taylor, L., Combe, J. \& 4 co-authors 2011 J. Geo. Res.,116, E00G05

Nakajima, M. \& Stevenson, D. J. 2015 Lunar \& Planet. Sci. Conf, 2770

Pieters, C., Goswami, J., Clark, R., \& 26 co-authors 2009, Science, 326, 568

Poston, M., Grieves, G., Aleksandrov, A., \& 4 co-authors 2012 Lunar \& Planet. Sci. Conf, 2801

Saal, A. E., Hauri, E. H., Van Orman, J. A., \& 4 co-authors 2008 Nature, 454, 192-195

Saal, A. E., Hauri, E., Van Orman, J. A., \& Rutherford, M. J. 2013 Science, 340, 1317-1320

Starukhina, L. V. 2006, Adv. Space Res., 37, 50-58 
Strycker, P., Chanover, N., Miller, C. \& 4 co-authors 2013 Nat. Comm., 4, 2620

Sunshine, J. M., Farnham, T., Feaga, L., \& 4 co-authors 2009 Science, 326, 565-568

Watson, K., Murray, B. C., \& Brown, H. 1961, J. Geophys. Res., 66, 3033-3045 